\title{
TRANSITIVE ACTIONS ON HIGHLY CONNECTED SPACES
}

\author{
VICTOR SCHNEIDER ${ }^{1}$
}

\begin{abstract}
Let $G$ be a compact, connected Lie group and $H$ a closed subgroup of $G$. It is shown that if $G / H$ is highly connected relative to $\operatorname{Rk}(G)-\operatorname{Rk}(H), G / H$ splits as a product of homogeneous spaces of simple Lie groups. This is used to show that the only transitive, effective actions on a large class of products of spheres are products of the known actions on the individual spheres.
\end{abstract}

1. Introduction. A homogeneous space is a differentiable manifold which admits a transitive, differentiable action by a compact, connected Lie group. A transitive action of $G$ on $X$ is called irreducible if no proper normal subgroup of $G$ already acts transitively on $X$. A natural problem is to classify all effective, transitive (or irreducibly transitive) differentiable actions by compact, connected Lie groups on a given homogeneous space. Homogeneous homotopy spheres were classified by Montgomery and Samelson [7], Borel [1] and Poncet [9] as follows: Any homogeneous homotopy sphere is standard, that is, has the usual differentiable structure; and, aside from a few low dimensional cases, the only irreducible actions are the standard ones. In [5] Hsiang and Su got similar results on Stiefel manifolds: For a large class of Stiefel manifolds, the only transitive actions are standard ones. One purpose of this paper is to classify irreducibly transitive actions on some highly connected spaces.

Any compact, connected Lie group $G$ can be uniquely expressed as follows:

$$
G=\bar{G} / N=\bar{G}_{1} \times \bar{G}_{2} \times \cdots \times \bar{G}_{s} / N
$$

where $\bar{G}_{i}$ is either $S^{1}$ or a simple, simply connected Lie group and $N$ is a finite normal subgroup of $\bar{G}$. If $G$ acts transitively on $X$, then $\bar{G}$ has a natural action on $X$ which may be represented as a translation on $\bar{G} / \bar{H}$. Let $\bar{H}_{i}=\bar{G}_{i} \cap \bar{H}$. If $\operatorname{Rk}(\bar{G})=\operatorname{Rk}(\bar{H})$, then $\bar{H}=\bar{H}_{1} \times \cdots \times \bar{H}_{s}$ and $X$ is diffeomorphic to $\bar{G}_{1} / \bar{H}_{1} \times \cdots \times \bar{G}_{s} / \bar{H}_{s}$. If $\operatorname{Rk}(\bar{H})>0$, it is not in general true that equality holds and so the action of $\bar{G}$ is not necessarily the product of the actions of the $\bar{G}_{i}$ on $\bar{G}_{i} / \bar{H}_{i}$. We show in this paper that if $\bar{G} / \bar{H}$ is

Received by the editors June 20, 1972.

AMS (MOS) subject classifications (1970). Primary 57E15.

Key words and phrases. Transitive action, $n$-connected, Lie group, Steenrod operations.

${ }^{1}$ The results presented here are part of a doctoral dissertation written at the University of Massachusetts under Professor L. N. Mann.

(c) American Mathematical Society 1973 
highly connected relative to $\operatorname{Rk}(\bar{G})-\mathrm{Rk}(\bar{H})$, then the action of $\bar{G}$ does split as a product action.

Let $G$ act transitively on $X=G / H$. Then the rank of $X$, denoted $\operatorname{Rk}(X)$, is defined as the difference in the rank of $G$ and $H$. This is well defined (independent of what Lie group is acting) and, in fact [5],

$$
\mathrm{Rk}(X)=-\sum_{i=1}^{\infty}(-1)^{i} \operatorname{dim}\left(\pi_{i}(X) \otimes Q\right),
$$

where $Q$ is the rationals. For positive integers $r$, we define $\phi(r)=4 r-1$ if $r \neq 1,2,4,5,8,9 ; \phi(r)=4 r+3$ if $r=1,2,5,9$; and $\phi(r)=4 r+7$ if $r=4,8$.

Theorem A (SPlitting TheOREM). Let $X$ be a homogeneous space having an irreducibly transitive action by a Lie group $G=\bar{G}_{1} \times \cdots \times \bar{G}_{s} / N$. If $\operatorname{Rk}(X)=r$ and $X$ is $\phi(r)$-connected, then $X$ is diffeomorphic to the product $\bar{G}_{i} / \bar{H}_{i} \times \cdots \times \bar{G}_{s} / \bar{H}_{s}$ and the $\bar{H}_{i}=\bar{G}_{i} \cap \bar{H}$ are simple and simply connected.

THEOREM B. If a homogeneous space $X$ of rank $r$ is a homotopy product of spheres with the dimension of each sphere greater than $\phi(r)$, then $X$ is diffeomorphic to that product of standard spheres. Moreover, any effective, irreducibly transitive action on $X$ is a product of the known actions on each sphere.

TheOREM C. Let $\mathrm{Rk}(X)=r$. Then, if $X$ is $[\max (39,4 r+3)]$-connected, $X$ is diffeomorphic to a product of standard Stiefel manifolds.

2. Preliminaries. All simple Lie groups $G$ are classified up to local isomorphism type. The classes are denoted $A_{r}, B_{r}, C_{r}, D_{r}$ (the classical groups) and $G_{2}, F_{4}, E_{6}, E_{7}, E_{8}$ (the exceptional groups). The following is a list of the degrees of the indivisible rational cohomology generators for each of the local isomorphism types and is also a list of the degrees of the infinite generators of the homotopy groups [3]:

$$
\begin{array}{ll}
A_{r}: & 3,5,7, \cdots, 2 r+1 \\
B_{r}: & 3,7,11, \cdots, 4 r-1 \\
C_{r}: & 3,7,11, \cdots, 4 r-1 \\
D_{r}: & 3,7,11, \cdots, 4 r-5,2 r-1 \\
E_{8}: & 3,15,23,27,35,39,47,59 \\
E_{7}: & 3,11,15,19,23,27,35 \\
E_{6}: & 3,9,11,15,17,23 \\
F_{4}: & 3,11,15,23 \\
G_{2}: & 3,11
\end{array}
$$

Therefore, the dimension of $H^{1}(\bar{G} ; Q)$ equals the number of $S^{1}$ factors of $\bar{G}$, and the number of indivisible generators in $H^{3}(\bar{G} ; Q)$ equals the number of simple factors of $\bar{G}$ which are simply connected. 
A straightforward spectral sequence argument will establish the following lemma for any coefficient group.

LEMMA 2.1. If $G / H$ is $n$-connected, the inclusion map $i: H \rightarrow G$ induces an isomorphism on cohomology through dimension $n-1$.

The next lemma follows immediately from Lemma 2.1 and the table.

LEMma 2.2. If $\bar{G} / \bar{H}$ is 4-connected, then $\bar{G}$ and $\bar{H}$ have the same number of $S^{1}$ and simple factors.

Lemma 2.3. Let $X=X_{1} \times X_{2}$, where $X_{i}$ is a compact, simply connected manifold of positive dimension. If $X$ has the homotopy type of a product of spheres and $H^{*}\left(X_{1} ; Z\right)$ has no more than one indivisible generator in any dimension, then $X_{1}$ has the homotopy type of a product of spheres.

Proof. Since there is no torsion in $H^{*}(X ; Z)$, we have $H^{*}(X ; Z) \simeq$ $H^{*}\left(X_{1} ; Z\right) \otimes H^{*}\left(X_{2} ; Z\right)$ and we may identify $H^{*}\left(X_{1} ; Z\right)$ with the subalgebra $H^{*}\left(X_{1} ; Z\right) \otimes 1$ of $H^{*}(X ; Z)$. So $X_{1}$ has the cohomology ring structure of a product of spheres. We will produce a product of spheres $Y$ and a map from $Y$ to $X$, which induces an isomorphism on $Z$-cohomology. Since the spaces $X_{1}$ and $Y$ are simply connected manifolds, the map must be a homotopy equivalence.

Let $f: \Pi^{s} S^{n_{j}} \rightarrow X$ be a homotopy equivalence and $p: X \rightarrow X_{1}$ be a projection. Suppose $1, u_{1}, \cdots, u_{k}$ is a set of indivisible generators of $H^{*}\left(X_{1} ; Z\right)$. Define $Y=\Pi^{k} S^{n_{i}}, n_{i}=\operatorname{deg} u_{i}$, and define $Y_{1}$ to be that factor of $\Pi^{s} S^{n_{j}}$ which consists of all the spheres whose dimension is $\operatorname{deg} u_{i}$, $i=1, \cdots, k$.

Now for each $i, p^{*}\left(u_{i}\right)$ is contained in a basis for the indivisible elements of $H^{q}(X ; Z), q=\operatorname{deg} u_{i}$. Since $f^{*}$ is an isomorphism, it takes a basis of indivisible elements to a basis of indivisible elements in $H^{q}\left(\Pi^{s} S^{n_{j}} ; Z\right)$. The submodule of these indivisible elements has a natural basis of $l \geqq 1$ generators coming from the $l$ spheres of dimension $q$ in the product; i.e., if $V$ is a generator in $H^{q}\left(S^{q} ; Z\right)$, a basis is formed by $V_{1}, \cdots, V_{l}$ which denotes the images of $V$ in $H^{q}\left(\Pi^{s} S^{n_{j}} ; Z\right)$ under the maps induced by projections on each factor. So we have $f^{*} p^{*}\left(u_{i}\right)=\alpha_{1} V_{1}+\cdots+\alpha_{l} V_{l}$. If each element of the basis containing $f^{*} p^{*}\left(u_{k}\right)$ is written as an integral combination of $V_{1}, \cdots, V_{l}$ and these coefficients are used as rows in a matrix, the matrix must have determinant \pm 1 since it expresses a change of basis in a free group. Expanding the matrix about the row $\alpha_{1}, \cdots, \alpha_{l}$, we get $\alpha_{1} A_{1}+\cdots+\alpha_{l} A_{l}= \pm 1$. Now we define two maps: Let $g_{i}: S^{q} \times \cdots \times$ $S^{q} \rightarrow S^{q} \times \cdots \times S^{q}, l$ factors, be a map which has degree $A_{1}, \cdots, A_{l}$ on respective factors and let $d_{i}: S^{q} \rightarrow S^{q} \times \cdots \times S^{q}, l$ factors, be the diagonal map. 
With these maps defined for each $i$, we have $g=g_{1} \times \cdots \times g_{k}: Y_{1} \rightarrow Y_{1}$ and $d=d_{1} \times \cdots \times d_{k}: Y \rightarrow Y_{1}$. Let $i: Y_{1} \rightarrow \Pi^{s} S^{n_{i}}$ be inclusion as a factor and identify $i^{*} V_{i}=V_{i}$. Hence we have the map pfigd: $Y \rightarrow X_{1}$, and since, for all $i$,

$$
\begin{aligned}
(p f i g d)^{*} u_{i} & =d^{*} g^{*} i^{*}\left(\alpha_{1} V_{1}+\cdots+\alpha_{l} V_{l}\right) \\
& =d^{*}\left(\alpha_{1} A_{1} V_{1}+\cdots+\alpha_{l} A_{l} V_{l}\right) \\
& =\left(\alpha_{1} A_{1}+\cdots+\alpha_{l} A_{l}\right) V \\
& = \pm V,
\end{aligned}
$$

the induced map is an isomorphism.

3. Splitting theorem. Let $G=G_{1} \times G_{2}$ act transitively on $X=G / H$. Let $H_{i}=G_{i} \cap H$. Following Montgomery and Samelson [7], we let $\Gamma_{i}$ be the image of $H$ under projection into $G_{i}$, and denote the product $\Gamma_{1} \times \Gamma_{2}$ by $\Gamma$. If $X$ is simply connected, then $H$ and, hence, $\Gamma_{i}$ and $\Gamma$ are connected. Let $x$ be the element of $X$ having isotropy subgroup $H$ and let $\Gamma(x)$ be the orbit of $x$ under $\Gamma$.

Lemma 3.1 (Montgomery And SAmelson) [7, P. 458]. If $G=G_{1} \times G_{2}$ acts transitively on a simply connected space $X$, then $\Gamma(x)=\Gamma_{i}(x), \Gamma / H$ is diffeomorphic to $\Gamma_{i} / H_{i}$, and $\Gamma_{i} / H_{i}$ is a compact, connected Lie group.

LEMMA 3.2. If there is an irreducibly transitive action of $G$ on a simply connected space $X$, then $G$ is semisimple.

Proof. Suppose $G$ is not semisimple. Then $\bar{G}$ can be written as $G_{1} \times G_{2}$ where $G_{1}=S^{1}$. Let $x \in X$ have isotropy subgroup $\bar{H}$. If $\Gamma_{1}=G_{1}$, then $X=G(x)=G_{2}\left(G_{1}(x)\right)=G_{2}\left(\Gamma_{1}(x)\right)$. But by Lemma 3.1, $\Gamma_{1}(x)=\Gamma_{2}(x)$, so $X=G_{2}(X)$, contradicting the fact that the action of $G$ is irreducibly transitive. If $\Gamma_{1} \neq G_{1}$, since $\Gamma_{1}$ is connected, $\Gamma_{1}$ is the identity, $e_{1}$, of $G_{1}$. So $\bar{H}=e_{1} \times H_{2}$ which implies that $X$ is not simply connected.

Proof of Theorem A. By Lemmas 2.2 and 3.2, we know that $\bar{G}$ and $\bar{H}$ are simply connected and have the same number of simple factors. Let $\bar{H}_{i}=\bar{G}_{i} \cap \bar{H}$. If $\bar{H}=\bar{H}_{i} \times \cdots \times \bar{H}_{s}$, there is nothing to prove. Suppose, to the contrary, that equality does not hold. Then clearly the $\bar{H}_{i}$ and their identity components $\bar{H}_{i}^{\circ}$ are normal in $\bar{H}$. But $\bar{H}_{1}^{\circ} \times \cdots \times \bar{H}_{s}^{\circ}=H^{\prime}$ can only be a proper normal subgroup of $\bar{H}$ if one of the $\bar{H}_{i}^{\circ}$ reduces to the identity. Since $H^{\prime}$ is normal, $H / H^{\prime}$ is a Lie group and $\Pi_{3}\left(\bar{H} / H^{\prime}\right)$ must have at least one infinite generator. Since $\Pi_{3}(\bar{H})$ has $s$ such generators, we see by the homotopy sequence for $H^{\prime} \rightarrow H \rightarrow \bar{H} \mid H^{\prime}$ that $\Pi_{3}\left(H^{\prime}\right)$ must have less than $s$ infinite generators and so must have less than $s$ factors. Hence, one of the $\bar{H}_{i}$ is finite. $\bar{G}$ can be rewritten as $G_{1} \times G_{2}$ where $G_{1}$ is the $\bar{G}_{i}$ having finite $\bar{H}_{i}$ and $G_{2}$ is the rest of the factors. Let $H_{i}=G_{i} \cap \bar{H}$. We will show that $\Gamma_{1}=G_{1}$. This will prove Theorem A by showing that the factor $G_{2}$ acts transitively. 
Since $H_{1}$ is finite, $\bar{H}$ is a finite covering of $\Gamma_{2}$ and thus $\operatorname{Rk}(\bar{H})=\operatorname{Rk}\left(\Gamma_{2}\right)$. Also $\quad r=\operatorname{Rk}(X)=\mathrm{Rk}(\bar{G})-\mathrm{Rk}(\bar{H})=\mathrm{Rk}(\bar{G})-\mathrm{Rk}\left(\Gamma_{2}\right) \geqq \mathrm{Rk}(\bar{G})-\mathrm{Rk}\left(G_{2}\right)=$ $\operatorname{Rk}\left(G_{1}\right)$. Since $\bar{G} / \bar{H}$ is $\phi(r)$-connected, we see from the exact homotopy sequence of the fiber bundle $\Gamma / \bar{H} \rightarrow \bar{G} / \bar{H} \rightarrow \bar{G} / \Gamma$, that $\pi_{q}(\bar{G} / \Gamma) \simeq \pi_{q-1}(\Gamma / \bar{H})$ for $q \leqq \phi(r)$. By Lemma $3.1, \Gamma / \bar{H}$ is homeomorphic to the compact, connected Lie group $\Gamma_{i} / H_{i}$, so $\pi_{q-1}(\Gamma / \bar{H})$ is finite whenever $q-1$ is even. Since $\bar{G} / \Gamma=G_{1} / \Gamma_{1} \times G_{2} / \Gamma_{2}, \pi_{q}\left(G_{1} / \Gamma_{1}\right)$ is finite for odd $q \leqq \phi(r)$. Hence for every infinite generator in $\pi_{q}\left(G_{1}\right), q \leqq \phi(r)$, there is an infinite generator in $\pi_{q}\left(\Gamma_{1}\right)$. This can be seen by examining the homotopy sequence for the principal bundle $\Gamma_{1} \rightarrow G_{1} \rightarrow G_{1} / \Gamma_{1}$. If $G_{1}$ is a classical group or one of the exceptional groups $G_{2}, F_{4}$, or $E_{6}$, all the infinite homotopy generators of $G_{1}$ are in odd dimensions less than or equal to $\phi\left(\operatorname{Rk}\left(G_{1}\right)\right) \leqq \phi(r)$. So $\Gamma_{1} \subset G_{1}$ and has at least as many infinite homotopy generators. Hence $\operatorname{Rk}\left(\Gamma_{1}\right)=$ $\operatorname{Rk}\left(G_{1}\right)$ and the infinite homotopy generators for $\Gamma_{1}$ and $G_{1}$ are in the same dimensions. A check of Borel and Siebenthal's classification of subgroups of maximal rank [4] shows that $\Gamma_{1}=G_{1}$. Some of the infinite homotopy generators for $E_{7}$ and $E_{8}$ occur above the bound $\phi(r)$; however, by checking the list of generators and [4], we easily see that $\Gamma_{1}$ and $G_{1}$ are equal even in these cases. This completes the proof.

As the proof illustrates, the variations of $\phi(r)$ from $4 r-1$ are for technical reasons to handle the exceptional groups. The condition that $X$ be $(4 r-1)$-connected is necessary as the following example shows: Let $\Psi_{1}: \mathrm{Sp}(r-1) \rightarrow \mathrm{Sp}(r)$ and $\Psi_{2}: \mathrm{Sp}(r-1) \times \mathrm{Sp}(n-r+1) \rightarrow \mathrm{Sp}(n)$ for some $n>2 r$ be the standard embeddings. Define the embedding $\Psi: \operatorname{Sp}(r-1) \times$ $\mathrm{Sp}(n-r+1) \rightarrow \mathrm{Sp}(r) \times \mathrm{Sp}(n)$ by $\Psi^{\prime}\left(g_{1}, g_{2}\right)=\left(\Psi_{1}\left(g_{1}\right), \Psi_{2}\left(g_{1}, g_{2}\right)\right)$. Let $G=$ $\operatorname{Sp}(r) \times \operatorname{Sp}(n)$ and $H=\operatorname{Im} \Psi$. Then $G$ acts transitively on $G / H$ by translation, $G / H$ is $(4 r-2)$-connected but not $(4 r-1)$-connected, and the action is not a product action.

4. Theorems B and C. To prove Theorem B we will need the following two lemmas.

LEMMA 4.1. Let X be a homogeneous space of rank $r$ having the homotopy type of a product of spheres. If the dimension of each sphere is greater than $\phi(r)$, no exceptional group $G$ can act transitively on $X$.

PROOF. By considering the homotopy exact sequence for the principal bundle $H \rightarrow G \rightarrow X$, one can see that it is impossible for $X$ to satisfy the conditions of the lemma while $G$ is exceptional. Each exceptional group must be checked separately; the necessary homotopy information may be found in [3], [6], [11].

LEMMA 4.2. If $X$ is a homotopy product of spheres with the dimension of each sphere greater than 7 and $G$ is a simple classical group acting transitively on $X$, then $X$ is diffeomorphic to a standard sphere. 
Proof. We will prove the lemma in the $S U$ case; the other cases are similar. The following information is needed [2] (similar information for the other cases may be found in [2], [10]):

$H^{*}\left(S U(n) ; Z_{2}\right)$ is an exterior algebra with generators $w_{2 j+1}, j=1, \cdots$, $n-1$, and $\operatorname{deg} w_{2 j+1}=2 j+1$. Also

$$
\begin{aligned}
S q^{2 i} w_{2 j+1} & =C_{i}^{j} w_{2(j+i)+1}, & & \text { for } i \leqq j, j+1 \leqq n-1, \\
& =0, & & \text { otherwise, }
\end{aligned}
$$

where $C_{i}^{j}$ is the binomial coefficient reduced modulo 2 .

By Lemma 2.2, $H$ must be simple; in fact, $H$ must be $S U(k)$. If $H$ were not $S U(k), \pi_{5}(G / H) \otimes Q \neq 0$. So we have the principal bundle $S U(k) \rightarrow$ $S U(n) \rightarrow X$. Hence $X$ must be at least 8-connected and therefore

$$
i^{*}: H^{*}\left(S U(n) ; Z_{2}\right) \rightarrow H^{*}\left(S U(k) ; Z_{2}\right)
$$

is an isomorphism up to $\operatorname{dim} 7$ by Lemma 2.1 . By the naturality of the Steenrod squares, we have that $i^{*}$ is an isomorphism up to dimension $2 k-1$ provided $2 k$ is not a power of 2 . If $2 k$ is a power of 2 and the isomorphism does not extend to that level, a spectral sequence argument will show that there exists a generator in $H^{2 k-1}\left(X ; Z_{2}\right)$ that maps onto $w_{2 k-1} \in H^{2 k-1}\left(S O(n) ; Z_{2}\right)$. But $S q^{2} w_{2 k-1} \neq 0$, so by naturality we have a nonzero Steenrod square in $X$. This is clearly impossible. Hence $X$ can only be the product of the spheres of dimensions $2 k+1,2 k+3, \cdots$, $2 n-1$. Examining the spectral sequence for $S U(k) \rightarrow S U(n) \rightarrow X$ we see that the generators in dimensions $2 k+1$ and $2 k+3$ must map onto corresponding generators in $H^{*}\left(S U(n) ; Z_{2}\right)$. This will again give $X$ a nonzero Steenrod square unless $n-2 \leqq k$. If $n-2=k, X$ has exactly two infinite homotopy generators. By the work of Oniščik [8], we see that $X$ must be the complex Stiefel manifold of 2 frames in complex $n$-space. By [5, Theorem 3.6], $X$ cannot be a product of two positive-dimensional manifolds. Hence $n-1=k$ and $X$ is diffeomorphic to $S^{2 n-1}=S U(n) / S U(n-1)$.

Proof of Theorem B. Suppose there is an irreducibly transitive, effective action of $G$ on $X$. Then, by the Splitting Theorem, $X$ is diffeomorphic to the product $\bar{G}_{1} / \bar{H}_{1} \times \cdots \times \bar{G}_{s} / \bar{H}_{s}$. Lemma 4.1 can be used to eliminate the exceptional groups as possible factors. Suppose $\bar{G}_{i}$ is a classical group, say $S U(n)$. Then, as in Lemma 4.2, $\bar{H}_{i}$ must be $S U(k)$ and $\bar{G}_{i} / \bar{H}_{i}$ has a nonzero Steenrod operation unless $k=n-2$ or $n-1$. But in these cases, Lemma 2.3 applies and $\bar{G}_{i} / \bar{H}_{i}$ is a homotopy product of spheres. This is impossible if $k=n-2$ [5]. Hence by Lemma 4.2 each $\bar{G}_{i} / \bar{H}_{i}$ is a standard sphere with an almost effective, transitive action of $G_{i}$. Since the action of $\bar{G}$ is the product action, $N=N_{1} \times \cdots \times N_{s}$, where $N_{i}=\bar{G}_{i} \cap N$. So $G=\bar{G} / N=G_{1} \times \cdots \times G_{s}$, where $G_{i}=\bar{G}_{i} / N_{i}$ acts transitively and effectively on $\bar{G}_{i} / \bar{H}_{i}$, a standard sphere. This completes the proof. 
EXAMPLE. $S O(8)$ acts transitively on $S O(8) / S O(6)$ which is diffeomorphic to $S^{6} \times S^{7}$. This shows that it is necessary to place some restrictions on the dimension of the spheres in order to get the action to split as a product action.

Proof of Theorem C. By the Splitting Theorem, $X$ is a product of homogeneous spaces each having a transitive action by a simple, simply connected Lie group. Let $X_{i}$ be one of the factors and let $G_{i}$ be the group acting on it. Since $X$ is at least 39-connected, the same arguments used in Lemmas 4.1 and 4.2 show that $G_{i}$ is not exceptional and, if $G_{i}$ is a $S U(n)$, $\operatorname{Spin}(n)$ or $\operatorname{Sp}(n)$, then the isotropy subgroup $H_{i}$ is a $S U(k), \operatorname{Spin}(k)$ or $\operatorname{Sp}(k)$, respectively. The high connectedness of $X_{i}$ forces the embeddings to be standard by [5, Theorem 2.4], and so each $X_{i}$ is a standard Stiefel manifold.

\section{REFERENCES}

1. A. Borel, Le plan projectif des octaves et les sphères comme espaces homogènes, C. R. Acad. Sci. Paris 230 (1950), 1378-1380. MR 11, 640.

2. _ - Sur l'homologie et la cohomologie des groupes de Lie compacts connexes, Amer. J. Math. 76 (1954), 273-342. MR 16, 219.

3. - Topology of Lie groups and characteristic classes, Bull. Amer. Math. Soc. 61 (1955), 397-432. MR 17, 282.

4. A. Borel and J. de Siebenthal, Les sous-groupes fermés de rang maximum des groupes de Lie clos, Comment. Math. Helv. 23 (1949), 200-221. MR 11, 326.

5. W. Y. Hsiang and J. C. Su, On the classification of transitive effective actions on Stiefel manifolds, Trans. Amer. Math. Soc. 130 (1968), 322-336. MR 36 \#4581.

6. M. Mimura, On the homotopy groups of the exceptional Lie groups, Conference on Algebraic Topology, University of Illinois, Urbana, Ill., 1968.

7. D. Montgomery and H. Samelson, Transformation groups of spheres, Ann. of Math. (2) 44 (1943), 454-470. MR 5, 60.

8. A. Oniščik, Transitive compact transformation groups, Mat. Sb. 60 (102) (1963), 447-485; English transl., Amer. Math. Soc. Transl. (2) 55 (1966), 153-194. MR 27 \#5868.

9. J. Poncet, Groupes de Lie compacts de transformations de l'espace euclidian et les sphères comme espaces homogènes, Comment. Math. Helv. 33 (1959), 109-120. MR 21 \#2708.

10. H. Scheerer, Homotopieäquivalente kompakte Liesche Gruppen, Topology 7 (1968), 227-232. MR 37 \#4833.

11. H. Toda, Composition methods in homotopy groups of spheres, Ann. of Math. Studies, no. 49, Princeton Univ. Press, Princeton, N.J., 1962. MR 26 \#777.

Department of Mathematics, University of Massachusetts, Amherst, MassaCHUSETTS 01002

Department of Mathematics, University of Southwestern Louisiana, LafayETTE, LOUISIANA 70501 